\title{
Drug interactions of oral anticoagulants
}

\section{Gayathri Anil, Pradhyumna Muraleedharan, Atiya Rehman Faruqui*}

Department of Pharmacology, St. John's Medical College, Bangalore, Karnataka, India

Received: 31 October 2020

Revised: 10 December 2020

Accepted: 11 December 2020

\section{*Correspondence:}

Dr. Atiya Rehman Faruqui,

Email: atiyadoc@gmail.com

Copyright: (C) the author(s), publisher and licensee Medip Academy. This is an open-access article distributed under the terms of the Creative Commons Attribution Non-Commercial License, which permits unrestricted non-commercial use, distribution, and reproduction in any medium, provided the original work is properly cited.

\begin{abstract}
Background: Oral anticoagulants (OACs) are the drugs of choice where long-term anticoagulation is needed due to convenience of dosing. But their use has potential for several drug interactions. Monitoring for potential interactions with timely management will decrease the risk of complications of anticoagulation.

Methods: We aimed to assess the presence of potential drug-drug interactions in patients on oral anticoagulants for various indications. Prescriptions of a cohort of patients on oral anticoagulants were analyzed. Potential drug interactions were identified using free software available at www.drugs.com and classified into major, moderate and minor types.

Results: Of the 135 patients in the study, 83 were males and mean age was $52.9 \pm 17.3$ years. Most commonly used OACs were vitamin K antagonists (VKAs) (80.0\%) followed by direct oral anticoagulants (DOACs) (20.0\%). Median number of concomitant medications per patient was 4 (IQR 3-6). A total of 307 potential interactions were identified in 121 patients with a median of 2 interactions per patient. Of the 56 patients who had potential for major drug interactions, $45(41.6 \%)$ were on VKAs and $11(40.7 \%)$ on DOACs had potential to develop major interactions. Using logistic regression model, significant predictors of major drug interactions were age $>60$ years (OR 2.50; 95\% CI 1.05-5.95 $\mathrm{p}=0.04$ ) and presence of venous thromboembolism VTE (OR 0.09; 95\% CI 0.02-0.55; $\mathrm{p}=0.01$ ).

Conclusions: This hospital-based study showed potential drug interactions with OACs. Age more than 60 years and presence of VTE were significant predictors of major interactions. Awareness of potential interactions and monitoring doses of OACs help to prevent complications of therapy.
\end{abstract}

Keywords: Oral anticoagulants, Drug interactions, Warfarin

\section{INTRODUCTION}

Oral anticoagulants (OACs) have become drugs of choice for conditions where long term anticoagulation is needed since they have the advantage of convenience of dosing. OACs include coumarin derivatives like warfarin sodium and nicoumalone which are vitamin $\mathrm{K}$ antagonists (VKAs) and direct oral anticoagulants (DOACs) like direct thrombin inhibitors (DTIs) such as dabigatran and factor $\mathrm{Xa}$ inhibitors which include apixaban, rivaroxaban and edoxaban.
The use of OACs, however, has potential for several drugdrug and drug-food interactions. OACs interact with substances which alter their uptake or metabolism or those which interfere with hemostasis, fibrinolysis or integrity of epithelial surface. Administration of OACs may be associated with risk of bleeding due to overanticoagulation or thrombosis due to under dosing. ${ }^{1}$

Majority of drug interactions with VKAs are pharmacokinetic and occur when administered along with drugs which inhibit or induce CYP450 enzymes, mainly CYP2C9 and CYP3A4 which are involved in VKA metabolism. $^{2,3}$ Warfarin despite being an effective 
anticoagulant, has certain limitations like narrow therapeutic index, variable dose-response and potential for interactions with various commonly used drugs. Warfarin interacts most commonly with CYP 450 inhibitors such as fluconazole, amiodarone, cotrimoxazole and metronidazole. ${ }^{4}$ The anticoagulant effect is measured by International Normalized Ratio (INR). For most conditions, a target INR of 2 to 3 is maintained.

Direct thrombin inhibitors such as dabigatran being a substrate for P-glycoprotein (P-gp) has interactions with drugs inhibiting or inducing P-gp. ${ }^{5}$ Dabigatran has product labeling instructions recommending to avoid use of $\mathrm{p}$-gp inducers in general and to restrict doses of $\mathrm{p}$-gp inhibitors like amiodarone, dronadarone, clarithromycin, ketoconazole, quinidine and verapamil. $^{4}$ Factor $\mathrm{Xa}$ inhibitors like rivaroxaban and apixaban show interactions with agents which affect both CYP450 and P-gp metabolism. ${ }^{6,7}$

Pharmacodynamic interactions with oral anticoagulants are a result of the additive bleeding effect. OACs show pharmacodynamic interactions with drug classes such as antiplatelets, non-steroidal anti-inflammatory drugs and serotonergic agents. Warfarin when used concomitantly with acetaminophen increases the INR suggesting a possible pharmacodynamic interaction. ${ }^{8}$ A prospective longitudinal study by Shalansky et al found that acetaminophen was associated with 1.5 times increased bleeding risk in warfarin treated patients. ${ }^{9}$ Orbit- AF registry found that of the 7347 patients with atrial fibrillation $(\mathrm{AF})$ who received $\mathrm{OACs}, 35 \%$ patients also received aspirin. There was a significant $53 \%$ increase in major bleeding and 52\% increase in hospitalizations for bleeding in patients who were on concomitant aspirin and OAC therapy compared to those on OAC monotherapy. ${ }^{10}$ As the number of prescriptions of DOACs increases, it becomes important to predict and manage potential drugdrug interactions, especially in resource limited settings like India. ${ }^{4}$

Use of multiple medications in patients who are on anticoagulants can increase the risk of drug interactions. ${ }^{11}$ There exist many different definitions of polypharmacy based on the number of drugs. Polypharmacy is defined as the use of multiple medications in a patient and is very common in patients with multi-morbidity. Patients who have 5 or more medications are classified as having major polypharmacy and 10 or more medications as having excessive polypharmacy. ${ }^{12}$

In this study, we aimed to evaluate the potential drug- drug interactions with anticoagulants and identify the predictors of major drug interactions, among patients receiving various oral anticoagulants in a tertiary care hospital.

\section{METHODS}

This study is part of a larger prospective observational study which had follow-ups at 3 and 6 months and which looked at the use and adherence to oral anticoagulants in in-patients. The study was carried out at St. John's Medical College Hospital, Bangalore from February 2016 to August 2017. Patients of both genders aged 18 years and above visiting the departments of vascular surgery and cardiology, who were prescribed oral anticoagulants for different indications were included in the study. We excluded patients who could not come for follow-up visits to the hospital.

Sample size estimation was based on adherence rate of $27.5 \%$ to oral anticoagulants in a study by Jae Hee Jim et al. ${ }^{13}$ Considering an estimated proportion of 0.28 , an alpha error of $5 \%$ and a confidence interval of $90 \%$, the sample size obtained was 217 patients. Taking a dropout rate of $20 \%$, final sample size estimated was 250 . Given the limitations of follow-up visits, a convenient sample size of 135 was taken for this study.

Data were analyzed to record the drug-drug interactions with the OACs using free software available at www.drugs.com. Online drug interaction software are free resources available for physicians and patients and has been reliably used in studies. ${ }^{14,15}$ Based on this software, the interactions were classified as major, moderate and minor. When the same patient had more than one type of interaction, we also noted the highest level of interaction in them.

\section{Ethics approval}

Institutional ethics committee (IEC), St. John's Medical College, Bangalore approved the conduct of the study.

\section{Statistical analysis}

We summarized baseline data of patients as means with standard deviations for continuous variables and frequencies with percentages, for categorical variables. Predictors of major drug interactions were studied using multivariate logistic regression analysis. A $p$ value $<0.05$ was considered significant for all tests. Data management was done using Epi Info software version 7 and analysis was done using SPSS version 20.

\section{RESULTS}

Of the total 135 patients, 83 subjects were males and mean age of the subjects was $52.9 \pm 17.3$ years (Table 1 ). Majority of the subjects $(74.8 \%)$ were 40 years and above. More than half $(54.8 \%)$ of the participants were from rural areas. The most commonly used OACs were the vitamin $\mathrm{K}$ antagonists, warfarin and nicoumalone (Table 2). Most common indications for use of OACs were deep vein thrombosis (DVT) (40.7\%) followed by AF (28.1\%). Majority $(97.2 \%)$ of the patients who were on warfarin were dosed at $2 \mathrm{mg}$ or above.

The median number of concomitant medications per patient was 4. Maximum number of concomitant drugs 
used in a patient was 14. In our study, almost half of the patients $(49.6 \%)$ had 5 or more concomitant drugs whereas $5.92 \%$ of the patients had 10 or more (Figure 2). Among patients who were on VKAs, $43.5 \%$ of the patients had major polypharmacy whereas among those on DOACs, $74.1 \%$ had major polypharmacy. Excessive polypharmacy was seen in $4(3.7 \%)$ patients taking VKAs and in 4 $(14.8 \%)$ patients on DOACs (Figure 2).

Table 1: Demographic characteristics $(n=135)$.

\begin{tabular}{|lll|}
\hline Variable & $\mathbf{N}(\%)$ \\
\hline Mean age in years (+SD) & $52.9 \pm 17.3$ \\
\hline \multirow{2}{*}{ Gender } & Male & $83(61.5)$ \\
\cline { 2 - 3 } & Female & $52(38.5)$ \\
\hline Residence & Rural & $74(54.8)$ \\
\cline { 2 - 3 } & Urban & $61(45.2)$ \\
\hline $\begin{array}{l}\text { Median no. of concomitant } \\
\text { drugs (IQR) }\end{array}$ & $4(3-6)$ \\
\hline Major polypharmacy $(\geq \mathbf{5}$ drugs) & $67(49.6)$ \\
\hline
\end{tabular}

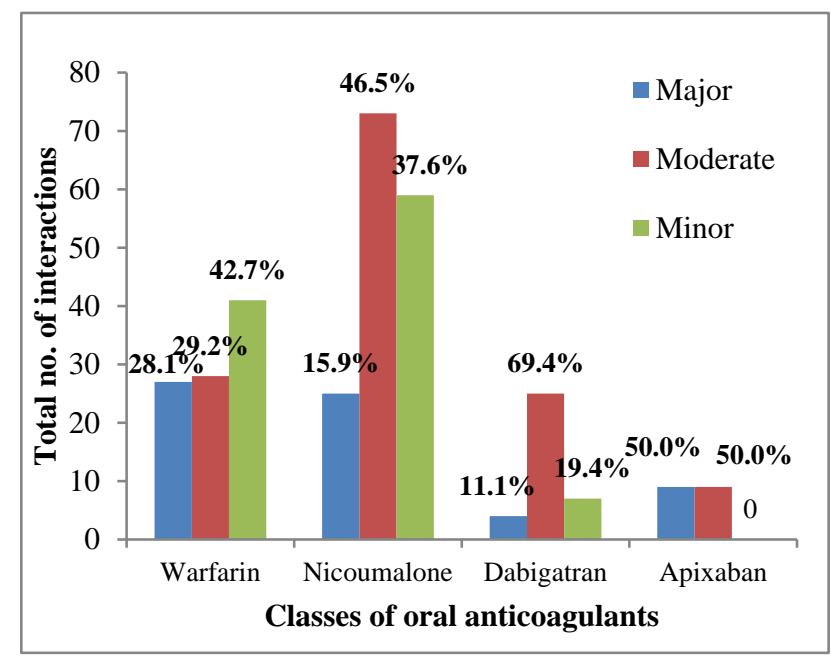

Figure 1: Interactions with oral anticoagulants $(\mathbf{n}=307)$.

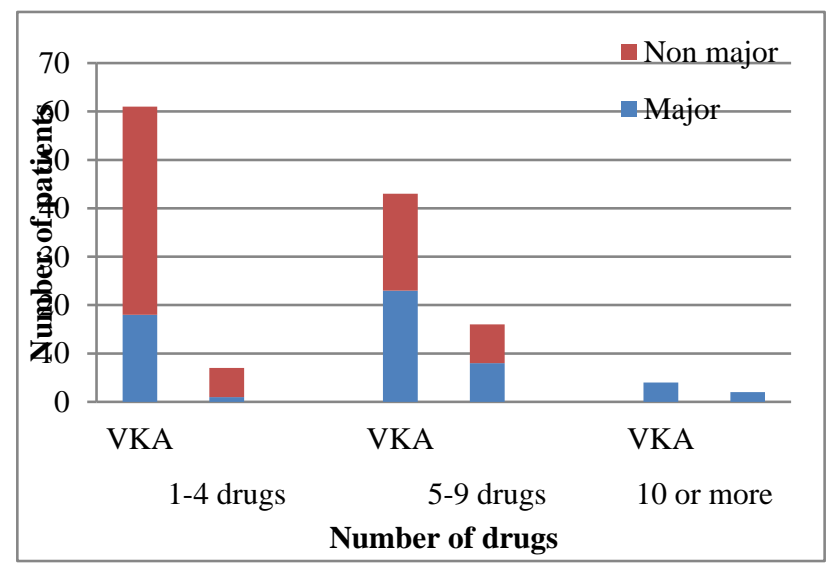

Figure 2: Polypharmacy and major drug-drug interactions.
A total of 307 potential interactions were identified in 121 patients in our study using the software. This reflects the possibility of more than one potential drug-drug interaction per patient. Median number of potential interactions per patient was 2 (IQR 1-3) (Table 3). Only 14 (10.4\%) patients in our study did not have any potential interactions. Of the 307 potential interactions identified, majority were moderate $(44.0 \%)$ followed by minor $(34.9 \%)$ and major (21.2\%). A breakup of the different types of interactions with individual drugs in given in Figure 1. Of the 56 patients who had potential to develop major interactions, majority were on nicoumalone $(44.6 \%)$ followed by warfarin $(35.7 \%)$ (Figure 3).

Table 2: Oral anticoagulant use $(n=135)$.

\begin{tabular}{|lll|}
\hline \multirow{2}{*}{ OACs } & & $\mathbf{N}(\%)$ \\
\hline \multirow{2}{*}{ DOACs } & Warfarin & $37(27.4)$ \\
\cline { 2 - 3 } & Nicoumalone & $71(52.6)$ \\
\hline \multirow{3}{*}{ Indications } & Dabigatran & $14(10.4)$ \\
\cline { 2 - 3 } & Apixaban & $13(9.6)$ \\
\hline & Atrial Fibrillation & $53(39.3)$ \\
\cline { 2 - 3 } & $\begin{array}{l}\text { Venous } \\
\text { thromboembolism }\end{array}$ & $74(54.8)$ \\
& $\begin{array}{l}\text { Arterial } \\
\text { thrombosis }\end{array}$ & $8(5.9)$ \\
\hline
\end{tabular}

Table 3: Interaction details $(n=135)$.

\begin{tabular}{|l|l|}
\hline Variable & $\begin{array}{l}\text { Total no. of } \\
\text { patients }\end{array}$ \\
\hline Total no. of interactions & 307 \\
\hline $\begin{array}{l}\text { Median no. of interactions } \\
\text { per patient }\end{array}$ & $2(1-3)$ \\
\hline $\begin{array}{l}\text { Total no. of patients with at } \\
\text { least one interaction }(\%)\end{array}$ & $121(89.6 \%)$ \\
\hline
\end{tabular}

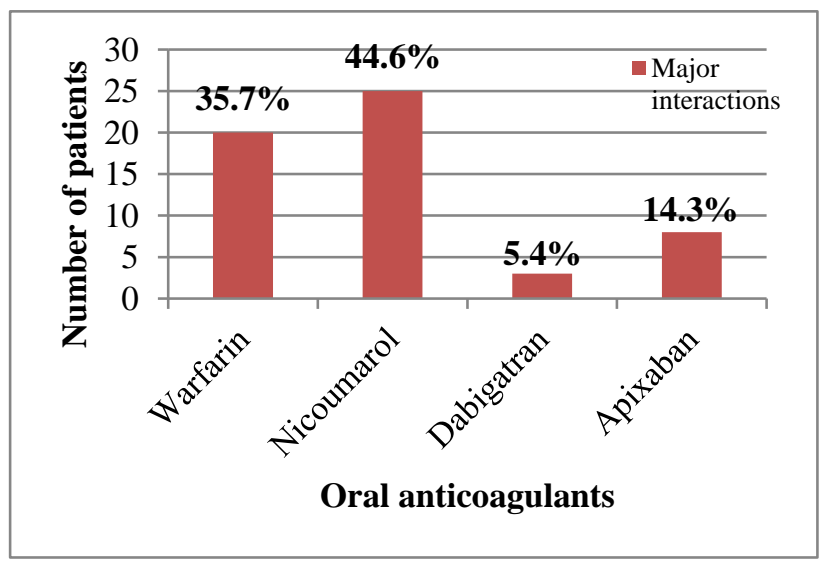

Figure 3: Major drug-drug interactions with individual OACs.

Of the total 66 major interactions found in our study, warfarin contributed to $40.9 \%$ (27 out of 66). Among patients using warfarin, drugs causing major interactions were aspirin, amiodarone and ciprofloxacin. $67.5 \%$ of the 
patients on warfarin had an international normalized ratio (INR) of 2 to $3 \mathrm{mg}$. Of the 71 patients using nicoumalone, 25 patients had major drug interactions, constituting $35.2 \%$ of the nicoumalone group. Among patients using nicoumalone, most common interactions were found with paracetamol, tramadol and aspirin. Details of interactions with Vitamin $\mathrm{K}$ antagonists are given in Table 4.

Table 4: Most common interactions with VKAs $(n=108)$.

\begin{tabular}{|lll|}
\hline \multirow{5}{*}{ Type of interaction } & Drugs & Number of patients, N $(\%)$ \\
\hline \multirow{5}{*}{ Major } & Aspirin & $37(34.3)$ \\
\hline \multirow{5}{*}{ Moderate } & Clopidogrel & $8(7.4)$ \\
\hline & Amiodarone & $4(3.7)$ \\
\hline & Ciprofloxacin & $1(0.9)$ \\
\hline \multirow{3}{*}{ Minor } & Pantoprazole & $23(21.3)$ \\
\hline & Tramadol & $20(18.5)$ \\
\hline & Glimepiride & $9(8.3)$ \\
\hline & Rosuvastatin & $3(2.7)$ \\
\hline & Amitryptiline & $3(2.7)$ \\
\hline & Atorvastatin & $24(22.2)$ \\
\hline & Paracetamol & $21(19.4)$ \\
\hline
\end{tabular}

Table 5: Most common interactions with DOACs $(\mathrm{n}=27)$.

\begin{tabular}{|lll|}
\hline \multirow{5}{*}{ Type of interaction } & Drugs & Total no. of patients, $\mathbf{N}(\%)$ \\
\hline \multirow{2}{*}{ Major } & Clopidogrel & $5(18.5)$ \\
\cline { 2 - 3 } & Aspirin & $5(18.5)$ \\
\hline \multirow{2}{*}{ Moderate } & Ibuprofen & $1(3.7)$ \\
\hline \multirow{2}{*}{ Minor } & Fondaparinux & $1(3.7)$ \\
\hline & Aspirin & $9(33.3)$ \\
\hline & Spironolactone & $9(33.3)$ \\
\cline { 2 - 3 } & Amiodarone & $4(14.8)$ \\
\hline
\end{tabular}

Table 6: Predictors of major drug-drug interactions.

\begin{tabular}{|llll|}
\hline Variable & Adjusted odd's ratio & $95 \%$ Confidence interval & P value \\
\hline Age $>$ 60 years & 2.50 & $1.05-5.95$ & 0.04 \\
\hline Urban residence & 2.30 & $1.02-5.21$ & 0.05 \\
\hline Atrial fibrillation & 0.39 & $0.06-2.37$ & 0.30 \\
\hline Venous thromboembolism & 0.09 & $0.02-0.55$ & 0.01 \\
\hline VKAs & 1.68 & $0.62-4.58$ & 0.31 \\
\hline Major polypharmacy & 1.70 & $0.74-3.90$ & 0.21 \\
\hline
\end{tabular}

*Multivariate logistic regression analysis

Among the DOACs, a total of 36 interactions were attributed to dabigatran, majority of which were of moderate category. Three of the fourteen patients $(21.4 \%)$ on dabigatran had major interactions. $61.5 \%$ of patients using apixaban had a major drug interaction. Among those using NOACs, most common drugs causing interactions were aspirin, clopidogrel and spironolactone. Details of the interactions with DOACs are given in Table 5.

Predictors of major drug interactions were analyzed using multivariate logistic regression analysis. Variables which had $p$ value of $<0.2$ in univariate regression were taken for multivariate analysis. $\mathrm{P}$ value of $<0.05$ was considered significant in multivariate regression. Age more than 60 years (OR 2.50; 95\% CI 1.05-5.95; $\mathrm{p}=0.04)$ and VTE (OR $0.09 ; 95 \%$ CI $0.02-0.55 ; \mathrm{p}=0.01)$ were significant predictors of potential major drug interactions. Major polypharmacy was not significantly associated with development of major interactions (OR 1.70; $95 \%$ CI 0.743.90; $\mathrm{p}=0.20)$ (Table 6).

\section{DISCUSSION}

In this study, a median of 4 drugs (IQR 3-7) were prescribed per patient and there was a median of 2 (IQR 13 ) interactions per patient. A total of 307 interactions were identified in 121 patients of which majority (44\%) were of moderate type. Major interactions were 65 in number 
(21.2\%) and minor constituted 107 (34.9\%). A similar study on drug-drug interactions with warfarin, done in 2014 in Ethiopia by Teklay et al reported a mean of $6.0 \pm 3.3$ drugs per patient with a mean of $3.2 \pm 2.0$ drug interactions per patient. The total number of drug interactions identified in their study with a sample size of 133 was 428 . The same study also reported the most common type of interaction as moderate type which accounted for $72.4 \%$. The proportion of patients with at least one drug interaction was $99.2 \%$. $^{3}$ This was more than that seen in our study which had $88.9 \%$ of patients with at least one potential drug-drug interaction. Another open cohort prospective study done in Brazil by Colet et al looked at drug interactions and adverse events in warfarin users who attended public health clinics. ${ }^{16}$ Colet et al reported the mean number of medications taken by their study population as $10 \pm 4$ which was higher than reported in other studies. Mean number of potential interactions per patient was $3 \pm 1.5$ which was also higher than in our study. They also reported that $97.1 \%$ of their population had interactions. ${ }^{16}$

The most common indication for OAC use in our study was DVT $(40.7 \%)$ followed by AF (28.1\%) which showed similarity with the previous study. ${ }^{3}$ The patient population in our study was older with a mean age of $52.9 \pm 17.3$ years than in the study by Teklay et al which reported a mean age of $40.8 \pm 17.6$ years. The mean age of the group of patients prescribed warfarin in our study was $53 \pm 17.6$ years. Teklay et al reported that in their study, more than $50 \%$ patients who were on warfarin were less than 40 years of age. ${ }^{3}$ In our study, the patients who were prescribed dabigatran were older (mean $63.6 \pm 18$ years) whereas the nicoumalone group was comparatively younger with a mean age of $49.8 \pm 15.6$ years. Our study had a male predominance with $65.1 \%$ being males whereas the study by Tekley et al had a female predominance $(58.9 \%)$. Colet et al reported the mean age of their population of warfarin users to be $64 \pm 14$ years with $55.1 \%$ female population. ${ }^{16}$

Polypharmacy plays an important role in development of drug interactions. A post hoc analysis of the ARISTOTLE trial which studied the treatment effect of apixaban versus warfarin reported rates of polypharmacy to be $76.5 \%$ in patients with $\mathrm{AF} .{ }^{17}$ The study found an increase in the number of drug interactions with increasing number of concomitant medications. However, in our study, on doing a logistic regression analysis, major polypharmacy was not found to be a significant predictor for developing major drug interactions.

The most dangerous interactions with coumarin anticoagulants are pharmacokinetic interactions involving P450 CYP2C9 which increase the anticoagulant effect and risk of bleeding. Pharmacodynamic interactions with coumarin anticoagulants occur due to synergism, competitive antagonism and an altered physiological control of vitamin $\mathrm{K}$. In our study warfarin had potential to cause major drug interactions with aspirin (34.3\%) and clopidogrel $(7.4 \%)$. Antiplatelet doses of aspirin and clopidogrel when given with warfarin increase the risk and intensity of bleeding. In a large retrospective case-control study done using the records from UK general practice research database from 2000 to 2005 , marked increase in gastrointestinal bleeding risk was found with concurrent use of aspirin and warfarin compared with either drugs used alone (rate ratio of 6.48 for combined therapy vs 1.39 for aspirin alone and 1.94 for warfarin alone). The study also found that acetaminophen use was significantly associated with increased gastrointestinal bleeding. ${ }^{18}$ ACC/AHA 2008 guidelines states that when warfarin is added to dual antiplatelet therapy (DAPT), the lowest effective INR of 2.0 to 2.5 should be targeted and the treatment should be for the shortest reasonable duration. However, use of warfarin with antiplatelet therapy (APT) is justified in patients in whom benefits outweigh risks of combined treatment, like in patients with prosthetic heart valves who are at high risk for thromboembolism.

In our study, $3.7 \%$ patients on VKAs were at risk of major interactions with amiodarone. Colet et al reported that $14.8 \%$ of the patients had potential major interactions with amiodarone. ${ }^{16}$ Amiodarone inhibits metabolism of both $\mathrm{R}$ and $\mathrm{S}$ enantiomers of a racemic mixture of warfarin and causes an increase in prothrombin time (PT), INR and bleeding risk. Warfarin when used concomitantly with amiodarone, may require a dose reduction by $25 \%$ to $65 \%$. INR monitoring should be done after one week of initiation of amiodarone therapy and thereafter once weekly until the INR is stable. If amiodarone therapy is withdrawn, a gradual increase in dosage of warfarin over the first few months may be warranted. ${ }^{4}$ Colet et al reported that $47.5 \%$ had major interactions with simvastatin. ${ }^{16}$ Our patients were not prescribed simvastatin, but $2.7 \%$ patients on rosuvastatin had potential for moderate interactions with VKAs.

In our study, DOACS were found to cause potential interactions with antiplatelet agents like aspirin, clopidogrel and diuretics like spironolactone. DOACs being substrates of gut mucosal efflux transporter pglycoprotein, interact with drugs which inhibit or induce pgp. The oral direct factor Xa inhibitors like rivaroxaban and apixaban are also partly metabolized by CYP3A4 enzymes and therefore interact with drugs inhibiting or inducing their metabolism. A meta-analysis of four warfarin/DOAC trials in AF patients reported that among more than 42,000 patients, $33.4 \%$ were on APT in addition to the anticoagulant. ${ }^{19}$ The same meta-analysis also showed that patients on a DOAC plus APT had a $33 \%$ increased rate of bleeding compared to those on DOAC alone without any additional benefit of thromboembolic event prevention. Warfarin and single or dual APT was shown to increase the risk of bleeding by 2 to 4 -fold. ${ }^{19}$

Interactions with DOACs are difficult to interpret because of its unknown magnitude. Empiric dose adjustments are not recommended with DOACs because patients can have variable responses. To avoid interactions between dabigatran and p-gp inhibitor, doses of the two drugs can be separated by 2 hours as dabigatran etexilate is a prodrug 
and separating its administration from the p-gp inhibitor will lead to its optimal absorption. This cannot be done with other DOACs because none of them are pro drugs. In a study done by Feng et al in adult AF patients, it was found that potential drug-drug interactions were significantly lower in those who switched from warfarin to DOACs. ${ }^{20}$

We found that patients above 60 years and presence of VTE were significant predictors of major drug interactions. However, the results of the multivariate analysis should be viewed with caution owing to the small sample size and single study centre. Study by Tekley et al has found that bleeding risk with warfarin was strongly associated with increased INR. ${ }^{3}$ Feng et al reported that the number of potential drug interactions and the proportion of days with potential drug interactions were significantly associated with risk of bleeding. ${ }^{20}$ In the excessive warfarin anticoagulation (EWA) study done in AF patients, excessive alcohol consumption and reduced renal function were the strongest predictors of excessive warfarin anticoagulation, defined as INR values $\geq 9$. Recent intake of antibiotic or antifungal drugs, recent hospitalization and outpatient visit were the other significant temporary predictors of excessive anticoagulation with warfarin. ${ }^{21}$ In our study, major polypharmacy was not a significant predictor for major drug interactions.

\section{Limitations}

Our study assessed only the potential drug interactions in patients on oral anticoagulants. We could not however assess the clinically observable responses in these patients. Following up the patients for clinically significant drug interactions with regular monitoring of INR values would help prevent complications of over or under anticoagulation. Also, none of our patients were prescribed rivaroxaban and hence rivaroxaban could not be assessed in our study. Because of the smaller sample size, our study was not statistically powered to assess certain other important factors which might have led to drug interactions, such as dose and duration of warfarin and INR values.

\section{CONCLUSION}

In this study, the most common drugs interacting with oral anticoagulants were found to be antiplatelet agents like aspirin and clopidogrel, anti-arrhythmics like amiodarone and statins. Management of potential drug-drug interactions with oral anticoagulants is complex and requires frequent INR monitoring, withdrawal of the interacting drugs, dosage adjustments of oral anticoagulants, use of reversal agents or replacement with drugs which have less potential for interactions.

\section{ACKNOWLEDGEMENTS}

Authors would like to thank to the staff of the departments of Pharmacology, Vascular Surgery and Cardiology and patients recruited for the study from the departments of Vascular Surgery and Cardiology.

Funding: No funding sources

Conflict of interest: None declared

Ethical approval: The study was approved by the Institutional Ethics Committee

\section{REFERENCES}

1. Di Minno A, Frigerio B, Spadarella G, et al. Old and new oral anticoagulants: Food, herbal medicines and drug interactions. Blood Rev. 2017;31(4):193-203.

2. Jacobs LG. Warfarin pharmacology, clinical management, and evaluation of hemorrhagic risk for the elderly. Clin Geriatr Med. 2006;22(1):17-32.

3. Teklay G, Shiferaw N, Legesse B, Bekele ML. Drugdrug interactions and risk of bleeding among inpatients on warfarin therapy: A prospective observational study. Thromb J. 2014;12(1):1-8.

4. Vazquez SR. Drug-drug interactions in an era of multiple anticoagulants: A focus on clinically relevant drug interactions. Blood. 2018;132(21):2230-9.

5. Scaglione F. New oral anticoagulants: Comparative pharmacology with vitamin $\mathrm{K}$ antagonists. Clin Pharmacokinet. 2013;52(2):69-82.

6. Eriksson BI, Quinlan DJ, Weitz JI. Comparative pharmacodynamics and pharmacokinetics of oral direct thrombin and factor $\mathrm{Xa}$ inhibitors in development. Clin Pharmacokinet. 2009;48(1):1-22.

7. Gnoth MJ, Buetehorn U, Muenster U, Schwarz T. In Vitro and In Vivo P-Glycoprotein Transport Characteristics of Rivaroxaban. J Pharmacol Exp Ther. 2011;338(1):372-80.

8. Lopes RD, Horowitz JD, Garcia DA, Crowther MA, Hylek EM. Warfarin and acetaminophen interaction: A summary of the evidence and biologic plausibility. Blood. 2011;118(24):6269-73.

9. Shalansky S, Pharm D, Lynd L, et al. Gingi Warfarine. 2007;27(9):1237-47.

10. Steinberg BA, Kim S, Piccini JP, Fonarow GC. Use and Associated Risks of Concomitant Aspirin Therapy with Oral Anticoagulation in Patients with Atrial Fibrillation: Insights from the ORBIT-AF Registry. Circulation. 2013;128(7):721-8.

11. Abdel-Aziz MI, Ali MAS, Hassan AKM, Elfaham TH. Warfarin-drug interactions: An emphasis on influence of polypharmacy and high doses of amoxicillin/clavulanate. J Clin Pharmacol. 2016;56(1):39-46.

12. Masnoon N, Shakib S, Kalisch-Ellett L, Caughey GE. What is polypharmacy? A systematic review of definitions. BMC Geriatr. 2017;17(1):1-10.

13. Kim JH, Kim GS, Kim EJ, Park S, Chung N, Chu SH. Factors affecting medication adherence and anticoagulation control in Korean patients taking warfarin. J Cardiovasc Nurs. 2011;26(6):466-74.

14. Kim BYB, Sharafoddini A, Tran N, Wen EY, Lee J. Consumer mobile apps for potential drug-drug interaction check: Systematic review and content 
analysis using the mobile app rating scale (MARS). JMIR mHealth uHealth. 2018;6(3):1-13.

15. Kheshti R, Aalipour M, Namazi S. A comparison of five common drug-drug interaction software programs regarding accuracy and comprehensiveness. J Res Pharm Pract. 2016;5(4):257.

16. Colet $\mathrm{C}$ de F, Amador TA, Heineck I. Drug Interactions and Adverse Events in a Cohort of Warfarin Users Attending Public Health Clinics. Int J Cardiovasc Sci. 2018;32(2):110-7.

17. Jaspers Focks J, Brouwer MA, Wojdyla DM, et al. Polypharmacy and effects of apixaban versus warfarin in patients with atrial fibrillation: post hoc analysis of the ARISTOTLE trial. Bio Med J. 2016;353:i2868.

18. Delaney JA, Opatrny L, Brophy JM, Suissa S. Drugdrug interactions between antithrombotic medications and the risk of gastrointestinal bleeding. Can Med Assoc J. 2007;177(4):347-51.

19. Kumar S, Danik SB, Altman RK, Barrett CD, Lip GY, Chatterjee S, et al. Non-Vitamin K Antagonist Oral Anticoagulants and Antiplatelet Therapy for Stroke
Prevention in Patients With Atrial Fibrillation. Cardiol Rev. 2016;24(5):218-23.

20. Feng X, Sambamoorthi U, Innes K, Castelli G, LeMasters T, Xiong L, et al. Predictors of Major Bleeding Among Working-Age Adults with Atrial Fibrillation: Evaluating the Effects of Potential Drugdrug Interactions and Switching from Warfarin to Non-vitamin K Oral Anticoagulants. Cardiovasc Drugs Ther. 2018;32(6):591-600.

21. Jaakkola S, Nuotio I, Kiviniemi TO, Virtanen R, Issakoff M, Airaksinen KEJ. Incidence and predictors of excessive warfarin anticoagulation in patients with atrial fibrillation - The EWA study. PLoS One. 2017;12(4):1-11.

Cite this article as: Anil G, Muraleedharan P, Faruqui AR. Drug interactions of oral anticoagulants. Int J Basic Clin Pharmacol 2021;10:70-6. 